\title{
Improving Antenatal and Postnatal Attendance: Beyond Theory to Practical Solutions
}

\author{
Kizito Uzoma Ndugbu ${ }^{1 *}$ and Elsie Chizoba Madukwe ${ }^{2}$ \\ ${ }^{1}$ Federal University of Technology, Nigeria \\ ${ }^{2}$ Department of Pediatrics, Elsie Chizoba Madukwe University of Port Harcourt Teaching Hospital, Nigeria \\ *Corresponding author: Kizito Uzoma Ndugbu, Federal University of Technology, Owerri, Nigeria. \\ To Cite This Article: Kizito Uzoma Ndugbu, Elsie Chizoba Madukwe, Improving Antenatal and Postnatal Attendance: Beyond Theory to Practical \\ Solutions. Am J Biomed Sci \& Res. 2019 - 5(4). AJBSR.MS.ID.000925. DOI: 10.34297/AJBSR.2019.05.000925.
}

Received: 紫September 13, 2019; Published: 眥 September 27, 2019

\begin{abstract}
An antenatal care that seem to thrive on the understanding of the pregnancy state as a potentially dangerous biomedical state, and so goes on to have its mechanisms mainly on surveillance is proving ineffective in developing countries. Despite the many reasons given for poor utilization of ANC, especially in developing countries; which has been the concentration of much research, the main problem appears to be one: the programme seems to be contextually at odds with the situations of pregnant women and their families, especially in low-income areas. This review offers practical ways for a practical solution to begin.
\end{abstract}

Keywords: Antenatal Care; Pregnant Women; Maternal and Child Health; Pregnancy Outcomes; Continuum of Care

\section{Introduction}

Antenatal care (ANC) or high-quality prenatal care is an important aspect of the reproductive maternal, newborn and child health continuum of care. Continuum of care is an expression that captures the perceived enduring nature of care for the pregnant mother and child, even after birth. It recognizes that safe childbirth is critical to the health of both the woman and the newborn child - and that a healthy start in life is an essential step to a sound childhood and a productive life [1]. The ANC provides the forum for women to be educated by health care providers about healthy pregnancy behaviours, danger signs of complications, breastfeeding and family planning; noticing and treating pregnancy-related pre-clampsia/ eclampsia; prompt referral to specialized care when needed; encourage the use of skilled birth attendants; and minimize the danger of mother - to - child transmission of viral diseases.

As it were, ANC exists to ensure a veritable gateway to health services during and after maternity care moments. It ideally serves as an avenue of screening, identifying and treating other chronic conditions. It is a necessary opportunity to overall health of mother and child that, it is recommended for a positive pregnancy experience [2]. However, the still fluctuating increase maternal child mortality arising natal and neonatal cases; especially in sub-Saharan Africa, have become a pointer to the underuse or non-use of the opportunities ANC avails. Beyond the literatures about the reasons for its underutilizations, practical solutions that are people-concerned-oriented appear to be the leeway.

\section{The burden of underutilization of the ANC}

As a major interventional strategy to ensure that both the mother and child remain healthy especially in low resource areas, timely and prompt attendance to the clinic providing these services are essential. This is however, far from being the case. ANC attendance has regrettably been low. As such, across countries and territories, 1 in 4 pregnant women attends no ANC care, and more $43 \%$ have given births without institutional or skilled assistance [1]. The fact that this goes on amidst a reported decline of global maternal mortality ratios in recent years [3] makes it more worrisome. The global picture may be beclouding of what obtains in low income settings, and in rural areas; and so, may close serious inquiry into evaluating how the intervention model fares side by side contexts. A pointer to this is what obtains in sub-Saharan Africa.

The underuse of ANC has been steady in sub-Saharan African. In terms of ANC attendants and institutional delivery, Nigeria (51\%) lags behind Ghana (78.2\%), Benin Republic (58.2\%), Lesotho (70.4\%), Liberia (78.1\%) and Zimbabwe (64.8\%) [4-6]. The underutilization of ANC in Nigeria is noticeably $46.5 \%$ as regards maternal child health interventional services, with a greater percentage in the rural areas, where there is $61.1 \%$ of such non-atten- 
dance. Again, in the North West zone of Nigeria, ANC attendance is placed at $41 \%$ while in the South East zone; it is $91 \%$. In terms of institutional delivery, the rural utilization is placed at $23 \%$ while urban records a $63 \%$ use. In the South West, the utilization is placed at $76 \%$ while the North West records $12 \%$ [7].

Poor antenatal care is an essential risk factor for adverse pregnancy outcomes among women [8]. This is why it is recently estimated that, each year more than 500,000 women between the ages of 15 and 49 die of causes related to pregnancy and child birth a leading cause of death among women of child bearing age [9], and to know that almost all maternal deaths occur in the developing world, and more than half occur in sub-Saharan Africa [10] makes poor ANC attendance and utilization risky.

The burden of the underutilization of antenatal care, institutional delivery and postnatal care service borders on constructs of language and personalization of care; power and relations; and health literacy. Taking women's dietary habits, health knowledge, economic power and cultural background is vital. So, women who do not attend antenatal care, or who do so late are more prone to preeclampsia, eclampsia and anaemia besides severe birth outcomes including preterm births, low birth and stillbirth $[11,12]$.

As has been the preoccupation of most studies in antenatal care, reasons have been adduced, and determinants identified as causative to the underutilization of ANC [13-15]. Such reasons as; cultural beliefs, age of mother, working status of the woman, education level, family income, religion, decision-making autonomy of the woman, place of residence, women's understanding and perception of the need for attendance [15-18].

Truth is, there are going to be more reasons not to attend ANC as long as we keep searching for them. A dilemma needs to be acknowledged here, and caution not to overstretch it, is essential. The dilemma is that the antenatal care services appear to thrive on the understanding of pregnancy state as a potentially risky biomedical state; and so, have mechanisms mainly on surveillance. The danger to this is that it immediately places its services at contextually at odds with the situations of pregnant women and their families, especially in low resource settings.

But then, pregnancy is not a normal life event. It is a medical condition requiring professional monitoring and supervision. A pregnant woman need not be sick to begin ANC [19]; she needs only be pregnant and to have given birth to attend ANC. As such, attending ANC is not just to diagnose sickness or treat sickness; but more also to promote healthy living of mother and child. This is an understanding that can be effective with incorporation.

\section{Underutilization of ANC: Way to Practical Solutions}

Surely, the antenatal care intervention strategy has been beneficial to some women and infants. It appears to have marginalized others too. It appears not to have taken the necessary survival de- cisions, practices, attitudes and cultural worldviews that are often usefully inherent in the local context into consideration. This is not making a lie to the obvious that some cultural beliefs and habits are detrimental to health, especially women's health. The concern here is doing more. Strategically incorporating culturally appropriate understandings of maternal care tailored to individual communities is key. Participatory programmes where local women and community leaders are actively engaged in the planning of local antenatal services can improve outcomes.

Initiate community mobilization: This will involve the use of neighbourhood health committees, local radio programs in local languages, local cultural settings and information, education and communication (IEC) materials target to the community to educate families especially women on the need to attend antenatal at the onset of pregnancy. This is to be reinforced by providing ITNs, pediatric follow-up visit, and vaccinations through ANC clinics as a way of attracting pregnant women to the clinics.

Community distribution of IPTp (Intermittent preventive treatment in pregnancy): The uncertainty among health workers about SP (sulfadoxine-pyrimethamine) administration for IPTp has led to a decline in expanding IPTp-SP coverage to prevent and treat malaria in pregnancy [20]. Here, villagers are mobilized by staff at the local ANC clinics to select women volunteers on correct provisions of IPTp. These volunteers go into the nooks and crannies of the villages, and report back to the health workers to turn in their records.

Increasing availability of ITNs: Part of the complaints of poor service at the ANC clinics was lack of materials [21]. There is need to strengthen procurement, storage and distribution systems to ensure a consistent supply of ITNs. This must be provided at every opportunity - through ANC clinics, during follow-up visits for under-fives, and during vaccination. Mobilized village volunteers can go from house to house for counselling and discussions with women and their families. They can equally help to check how the ITNs are properly been used.

Advocacy: Part of the problem of ANC utilization harped on charges at the ANC \{folic acid, iron supplements, ANC records, cards and delivery preparation\}[22,23]. Making them free will bring about a healthy behaviour as regards ANC utilization. Also, part of advocacy will be to ensure routine monitoring. The current ANC cards and registers need to be revised so as to include places to mark when a person receives her IPTp-SP doses or is given ITNs. This is to ensure easier reporting on IPTp indicators and program progress.

\section{Conclusion}

Maternal mortality in the developed nations has been considerably reduced. But the story is not the same in the developing nations. Good quality evidence is scarce as regards how integrating 
antenatal care strategy can fulfil its goal among the developing nations of the world. It is no gainsaying that as long we continue to jettison the incorporation of the understandings of maternal care tailored to individual communities and circumstances, the ANC intervention aims remains unachieved in developing countries; especially sub-Saharan Africa, Nigeria particularly. Practical solutions are the way to go let us make ANC community participatory.

\section{References}

1. (2010) World health report 2005 - make every mother and child count.

2. (2016) WHO, Recommendations on antenatal care for a positive pregnancy experience.

3. Hogan MC, Foreman KJ, Naghavi M, Ahn SY, Wang M (2010) Maternal mortality for 181 countries, 1980-2008: a systematic analysis of progress towards millennium development goals 5. Lancet 375(9726):16091623.

4. (2019) USAID from the American People, STATcompiler the DHS Program.

5. Adewuyi EO, Auta A, Khanal V, Bamidele OD, Akuoko CP, et al. (2018) Prevalence and factors associated with underutilization of antenatal care services in Nigeria: a comparative study of rural and urban residences based on the 2013 Nigeria demographic and health survey. PLoS ONE 13(5): e0197324.

6. Lawn JE, Kerber K (2006) Opportunity for Africa's newborns: practical data, policy and programmatic support for newborn care in Africa. Geneva: WHO on behalf of PMNCH, UNFPA, UNICEF, USAID, WHO.

7. Dahiru T, Oche MO (2015) Determinants of antenatal care, institutional delivery and postnatal care services utilization in Nigeria. The Pan Afri Med J 21:321.

8. (2003) Antenatal care in developing country: an analysis of trends, levels and differentials, WHO.

9. Hill K, AbuZahr C, Wardlaw T (2001) Estimates of maternal mortality for 1995. Bull World Health Org 79(3): 182-193.

10. Manna PK, De D, Ghosh D (2011) Knowledge, attitudes and practice for antenatal care and delivery of the mothers of tea garden in Jalpaiguri and Darjeeling districts, West of Bengal. Natio J Comm Med 2: 4-8.
11. Tuladhar H, Dhakal N (2011) Impact of antenatal care on maternal and perinatal outcome: a study of Nepal medical college teaching hospital. NJOG 6(2): 37-43.

12. Abbas AM, RabeeaM, Abdel Hafiz HA, Ahmed NH (2017) Effects of irregular antenatal care attendance in primiparas on perinatal outcomes: a cross sectional study. Proceedings in Obst \& Gyne 7(2): 2.

13. Onah HE, Ikeako LC, Iloabuchie GC (2006) Factors associated with the use of maternity services in Enugu, southeastern Nigeria. Soc Sci Med 63 (7): 1870-1878.

14. Prata N, Ejembi C, Fraser A, Shittu O, Minkler M (2012) Community mobilization to reduce postpartum haemorrhage in home births in northern Nigeria. Soc Sci Med 74(8): 1288-1296.

15. Ogunba BO, Abiodun OB (2017) Knowledge and attitude of women and its influence on antenatal care attendance in southwestern Nigeria. J Nutr Health Sci 4(2): 207.

16. Regina K, Madison J (2009) Contemporary childbirth practices in Nepal: improving outcomes. Br J Midwifery 17(6): 382-87.

17. Gcaba R, Brookes HB (1992) The unbooked maternity patient in an academic hospital in Durban. Curationis 15(3): 43-47.

18. Atuyambe L, Mirembe F, Johanson A, Kirumira EK, Faxelid E (2009) Seeking safety and empathy: adolescent health seeking behaviour during pregnancy and early motherhood in central Uganda. J Adolesc 32(4): 781-796

19. Chowdhury AM, Mahbub A, Chowdhury AS (2003) Skilled attendance at delivery in Bangladesh: an ethnographic study. Research Monograph Series BRAC p. 22.

20. (2019) World Health Organisation.

21. Amooti Kaguna B, Nuwaka F (2000) Factors affecting choice of delivery sites in Rakai district of Uganda. Soc Sci Med 50(2): 203-213.

22. Abrahams N, Jewkes R Muo Z (2001) Healthcare seeking practices of pregnant women and the role of the midwife in Cape Town. SA J Midwifery Women's Health 46(4): 240-47.

23. Grossmann Kendall F, Filippi V, DeKonnick M, Kanhonou L (2001) Giving birth in maternity hospitals in Benin: testimonies of women. Reprod Health Matters 9(18): 90-98. 